\title{
Nonlinear optical spectroscopy of collagen transformation
}

\author{
V. Shcheslavskiy ${ }^{1}$, G. I. Petrov ${ }^{1}$, S. Saltiel ${ }^{2}$, A. Boyko ${ }^{3}$, V. Y. Petrussevich ${ }^{1}$, Y. M. Petrussevich ${ }^{3}$, G. \\ P. Petrova ${ }^{3}$, V. V. Yakovlev ${ }^{1 *}$ \\ ${ }^{1}$ Department of Physics, University of Wisconsin - Milwaukee, P. O. Box 413., Milwaukee, WI \\ 53201, USA \\ ${ }^{2}$ Faculty of Physics, Sofia University, Bulgaria \\ ${ }^{3}$ Physics Department, Moscow M. V. Lomonosov State University, Moscow 119992, Russia
}

\begin{abstract}
We report on the novel application of nonlinear optics to study molecular assemblies. By using the third-harmonic generation in solution we were able to determine both the third-order nonlinear susceptibility of collagen and the size of fibrils. The developed approach also helped us in revealing the helix-coil transformation of collagen in solution.
\end{abstract}

Keywords: third-harmonic generation, collagen, transformation, nanoscale

\section{INTRODUCTION}

Collagen is one of the most important proteins in living organisms [1]. Collagen molecules are structural macromolecules of the extracellular matrix that include in their structure one or several domains having a characteristic triple helical conformation. These helical and suprahelical properties of collagen are responsible for its ability to generate strong nonlinear optical signals [2].

Nonlinear optical methods based on second harmonic generation are already used for microscopic imaging of collagen [3]. In spite of the fact that there has been a comprehensive research on nonlinear optics of collagen, a number of important questions were left unanswered.

What is the relationship between the structure of collagen and its nonlinear optical properties? It is important to know that to better understand structural changes which can happen during processes of thermal denaturation, glycation, enzymatic cleavage and other processes, which are specific for pathophysiologic conditions in biological tissues. Comparison of the results on nonlinear susceptibility of the collagen at different levels of organization (monomer-fibrilfascicle) may provide very important information on polarity of the biological tissues in general. There are several advantages of using high harmonic generation signals for analysis of the collagen. First, it requires no fluorophore labeling of the molecules. Second, second and third harmonic generation can serve as sensitive probe of molecular orientation. This is especially important when studying how single molecules assemble in supramolecular structures [4]. Finally, nonlinear behavior tends to be a more sensitive indicator of structural changes in a molecule, than linear one [5].

The most detailed studies of nonlinear optical properties of collagen were conducted by Freund et al [6] who used second-harmonic generation (SHG) microscopy for determination of second-order nonlinear susceptibility of the collagen fibrils. They measured it to be one-half of the crystalline quartz and found that the rat-tail tendon exhibits macroscopic polar order. Since SHG is polarization dependent, it may provide information about fiber orientation [7].

There was also research on third harmonic generation in biological tissues $[8,9]$. The third harmonic conversion efficiency from skin was estimated to be about $10^{-9}$ and smaller than SHG conversion efficiency by two orders of magnitude. In these experiments the wavelength of the third harmonic was in the absorption region of collagen and the power densities were quite high $\left(0.5 \mathrm{~J} / \mathrm{cm}^{2}\right)$, making difficult the quantitative interpretation of the results.

*Email: yakovlev@uwm.edu; Phone: +1-414-229-3978; Fax: +1-414-229-5589 
All the nonlinear optical studies of collagen have been performed with either the biological tissues, containing collagen fibrils [8,9], or with fascicles deposited on the slides [10]. In first case, there are a lot of other factors influencing SHG (for instance, elastin can contribute to SHG from skin). In the latter case, measurements were done at the conditions far from physiological conditions. In this report we use microscopic arrangement for third-harmonic generation in solution to determine not only the nonlinear susceptibility of collagen, but also to estimate the size of collagen fibrils.

\section{THEORETICAL BACKGROUND}

To determine the nonlinear optical susceptibility of collagen in solution we use a recently developed technique [12], which uses the third-harmonic generation on two consecutive interfaces (air-glass, and glass-solution). While the power

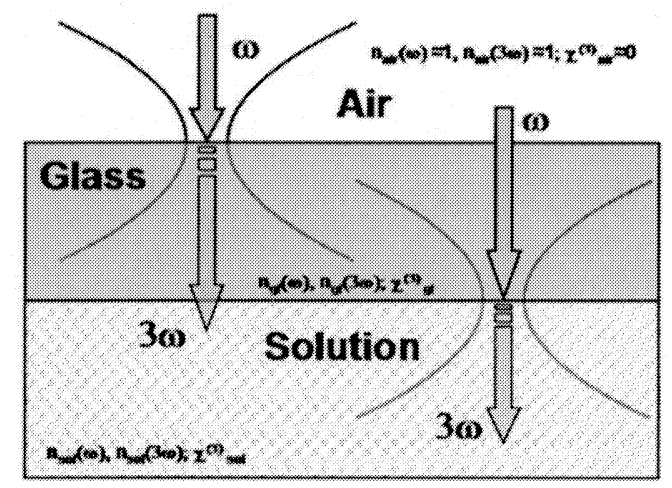

Figure 1. Schematic illustration of the technique of nonlinear susceptibility measurements based on the thirdharmonic generation on the interface.

of the third harmonic generated from a focused beam vanishes in homogeneous medium, it is rather strong on the interface between two media due to the sharp change of either refractive index or nonlinear optical susceptibility. This generated third-harmonic power can be described in terms of refractive indices of two media for both fundamental and third-harmonic waves, and nonlinear optical susceptibilities of both media. By simultaneous measurements of the thirdharmonic signals generated on two interfaces (see Fig. 1), we can exclude the incident intensity from the equation, which

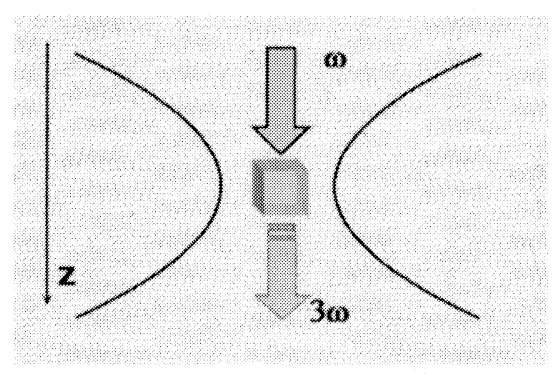

Figure 2. Third-harmonic generation from a small inhomogeneity in solution.

sets the relationship between the optical constants between of three media [12]. Assuming the knowledge of optical constants for two media (air and glass), and refractive index of solution under study, the nonlinear optical susceptibility, 
$\chi^{(3)}$, of solution can be easily extracted by solving a simple algebraic equation. The good thing about this technique is that it is applicable for $\chi^{(3)}$ measurements of highly scattering and highly absorbing solutions, and is almost insensitive to the mode quality of the incoming beam [12].

In this work, we extend our previous work and consider the generation of the third harmonic from a medium, which has some nanoscale homogeneities. In principle, this system can be treated the same way as the previous one with the assumption of two interfaces rather than one. We write Maxwell's equations for the electric field of the third-harmonic, and in a slow varying envelope approximation one can get [13]:

$$
2 i k_{3} \frac{\partial E_{3}}{\partial z}+\nabla_{T}^{2} E_{3}=-\frac{\pi \omega_{3}^{2}}{c^{2}} \chi^{(3)}\left(-3 \omega_{1}, \omega_{1}, \omega_{1}, \omega_{1}\right) E_{1}^{3} \exp [-i \Delta k z]
$$

where $E_{1}, E_{3}$ are the electric field, and $k_{1}, k_{3}$ - are the wavevectors for the of the incident and third-harmonic waves,

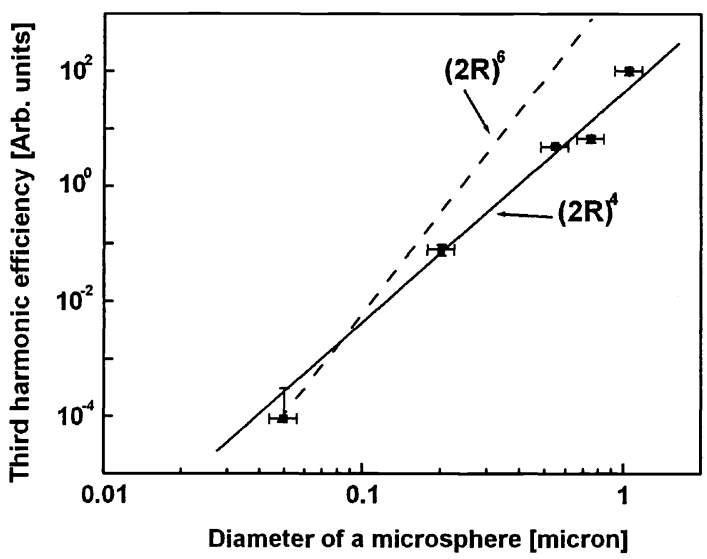

Figure 3. The power of the third harmonic generated from a microspheres as a function of its diameter. Squares - experimental data, the solid line shows theoretical fit with the fourth power dependence, the dashed line shows for illustration sixth power dependence.

respectively, $\Delta k=k_{3}-3 k_{l}$ is the mismatch wavevector, $\mathrm{z}$ - is the space coordinate along the wave propagation (see Fig. 2). By integrating Eq. (1) along the direction of propagation, noting the change of both the refractive indices and the nonlinear susceptibility in different media, we get a non-vanishing value for the power of the third-harmonic. For a spherically shaped inhomogeneity, whose size is significantly less than the beam waist, we can get a fourth power dependence of the generated third harmonic on the size of a microsphere. Moreover, the generated third harmonic power depends only on the size of microspeheres and the nonlinear optical susceptibility of its material. It allows us to extract the information on both the size of inhomogeneities and their nonlinear susceptibility from two independent measurements (i.e. by measuring the power of the third harmonic generated on the interface glass/solution, and the power of the third-harmonic generated inside the solution) by solving a set of algebraic equations.

\section{EXPERIMENTAL RESULTS}

\subsection{Testing the theory predictions}

In the previous section we made a rather strong statement about the third-harmonic behavior in the presence of microscopic and nanoscopic inhomogeneities. To be able to test these theoretical predictions we use polystyrene and 
silica nanospheres of various diameters and measure the power dependence of the third-harmonic power generated by a

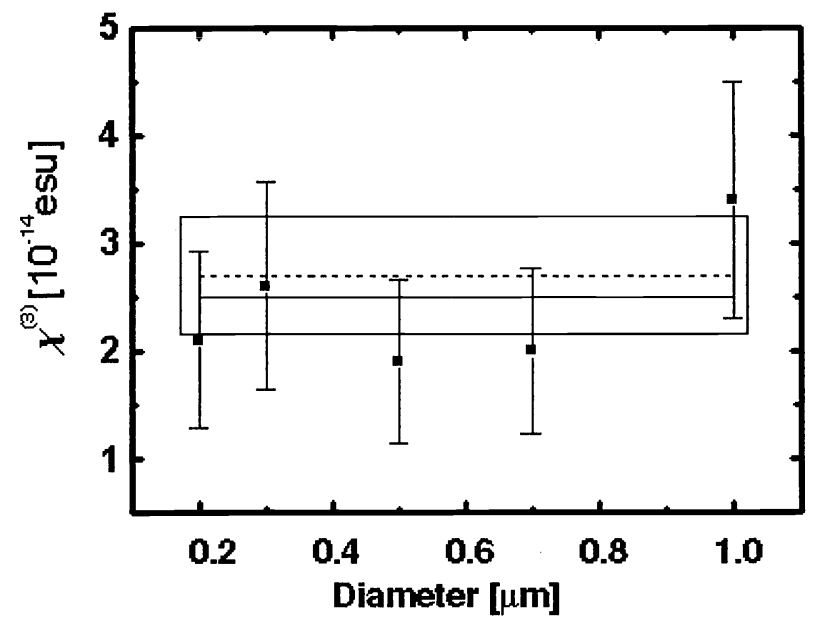

Figure 4. Third-order nonlinear susceptibility as a function of spheres' diameter. The solid line represents the value of $\chi^{(3)}$ for fused silica from literature [14]. The dashed line $-\chi^{(3)}$ from surface measurements. The solid rectangle represents the error of $\chi^{(3)}$ from volume measurements.

single microsphere as a function of its diameter. Within the accuracy of our measurements and the accuracy of independent measurements of microspheres' diameters, we find this dependence in an excellent agreement with the theoretically predicted one (see Fig. 3). Moreover, we are able to determine the nonlinear susceptibility of the known material (fused silica), from which one set of microspheres is made. These measurements are shown in Fig. 4 and show both the self-consistency of our "surface" and "bulk" measurements, and an excellent agreement with the previously reported data on fused silica [14].

The above findings give us a solid confirmation of our theoretical predictions. They also showed that features as small as 50-nm can be easily detected and characterized using third-harmonic measurements.

\subsection{Nonlinear spectroscopy of collagen}

Collagen type I is obtained from calf skin and supplied by ICN Biomedicals. Following the recipe [15], we dissolve the protein in $0.04 \mathrm{M}$ acetic acid solution with a concentration of about $10 \mathrm{mg} / \mathrm{ml}$. Then this concentrate is used for adding to pure $0.04 \mathrm{M}$ acetic acid solution in experiment. The $\mathrm{pH}$ of the solution is 4.8 .

We use femtosecond Cr:forsterite laser for our studies, for which both the fundamental wavelength and the thirdharmonic wavelength lie in a relatively non-absorbing spectral region of collagen. We vary the concentration of solution by slowly increasing it and measure both the surface (on the glass/solution interface) and the bulk (at least $10 \mathrm{Rayleigh}$ distances from the nearest interface) contribution to the third-harmonic power with respect to the power of the thirdharmonic generated on the first interface (air/glass) [16]. These dependences are shown in Fig. 5. They can be divided into three regions. In the first region, there is a steady growth of the third-harmonic power, which shows that the probability of finding collagen in the focal volume increases with concentration (region I). However, at a certain concentration level there is a sudden jump of the third-harmonic power (region II), and than - a sudden drop (region III) to the level beyond the expected level of the third harmonic power if there were no previous peculiar. We decided to use our theory to calculate both the nonlinear susceptibility of collagen, and its estimated size as a function of concentration. These calculated values are presented in Fig. 6, and they show quite an interesting effect. First, let us look at the size of molecular formation of collagen (see Fig. 6b). It shows a rather dramatic increase in size before reaching the critical concentration value, and than - an unexpected drop. It could be explained in terms of formation bigger fibrils in solution, 
which become unstable beyond the certain size and undergo a transformation from helix to coil structure. On the other

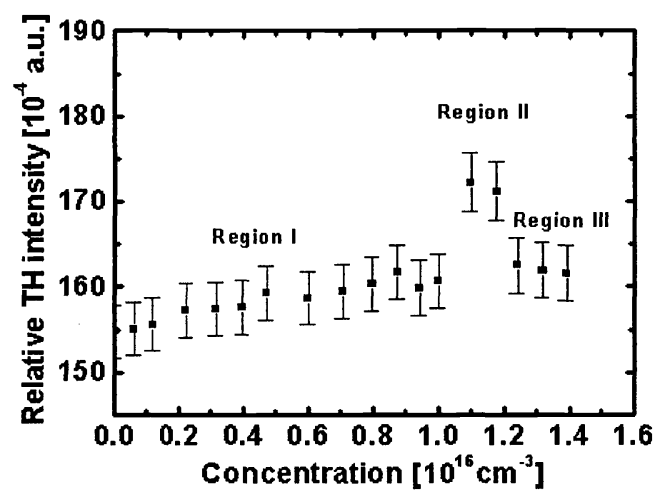

a)

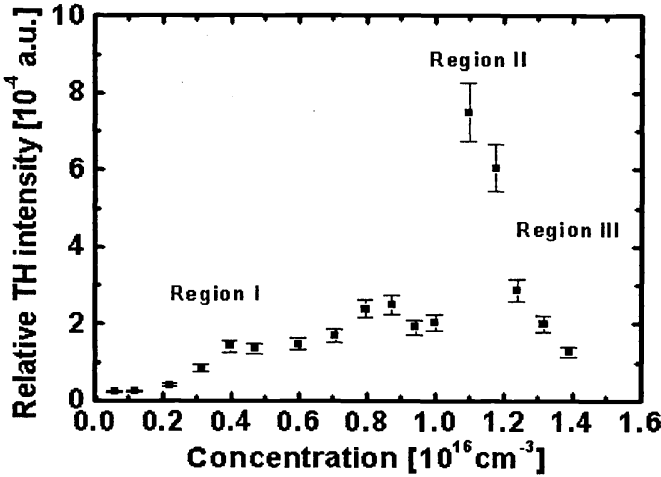

b)

Figure. 5. The relative third harmonic power generated on the glass/solution interface (a) and the relative third-harmonic power generated in the volume of solution (b) as a function of collagen concentration in acetic acid $(0.04 \mathrm{M})$ solution $(\mathrm{pH}=4.8)$.

hand, the nonlinear optical susceptibility remains almost unchanged until the concentration reaches its critical value (see Fig 6a), than increases and than drops again below its original value. This behavior of nonlinear susceptibility fits the described above scenario.

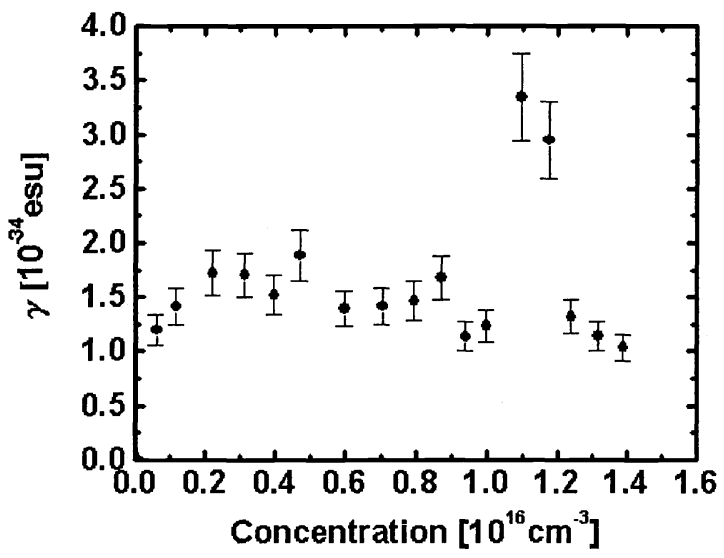

a)

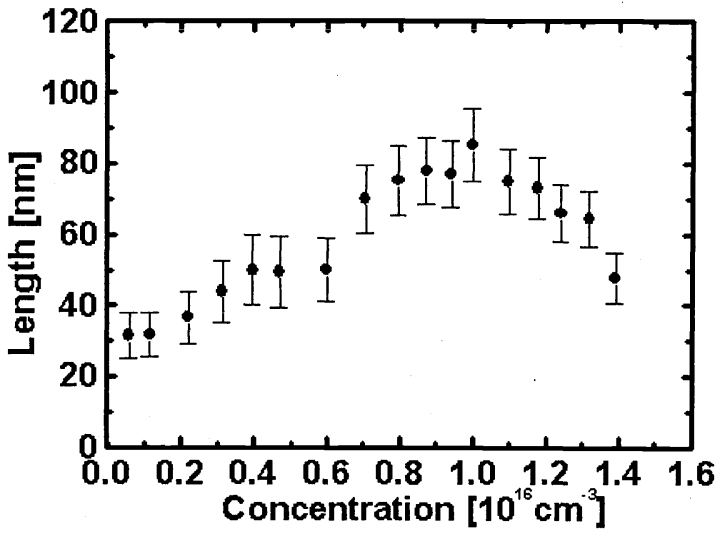

b)

Figure 6. (a) The measured value of second hyperpolarizability $\langle\gamma\rangle_{\text {col }}$ as a function of collagen molecules' concentration in solution. (b) The estimated characteristic sizes of the supramolecular assemblies in collagen solution.

However, in order to describe what is happening with collagen in solution upon the increase of its concentration we have to perform a series of other independent measurements. Below we provide a short summary of those studies.

Hyper Rayleigh scattering measurements also show a significant variation of nonlinear second-order susceptibility just near the critical concentration.

NMR measurements have revealed that molecular structure shows no substantial changes in the region of low collagen concentrations. Above the critical concentration, there is a substantial change of structure. However, the exact interpretation of these spectra are not possibly because of their complexity and the size of collagen molecule. 
Linear absorption measurements show a gradual change of absorption, with some features in the vicinity of the critical concentration.

Linear CD absorption measurements reveal a dramatic variation near the critical concentration, manifesting that in fact the helix-coil transformation does take place at this concentration.

Finally, light scattering experiments confirm the increase of the overall size of molecular formations of collagen and show some increase in the size of collage assemblies above the critical concentration.

From all these findings, we can conclude that with the increase of collagen concentration in solution its fibrils increase in size. When the size of fibrils reaches its critical value the structure becomes unstable, which results in the increase of its third-order polarizability. Further increase of collagen concentration leads to the helix-coil transformation.

\section{CONCLSUION}

We demonstrate for the first time that third-harmonic measurements can provide valuable information not only about the nonlinear polarizability of molecules in solution, but also about the size of molecular formations. The developed theory of the third-harmonic generation from nano-sized inhomogeneities was experimentally confirmed for a model system and successfully applied to study collagen fibrils formation in solution.

\section{ACKNOWLEDGEMENTS}

The author would like to gratefully acknowledge Prof. R. D. Heathcote (Department of Biology, University of Wisconsin - Milwaukee, USA), and A. V. Sklyarov (Department of Physics and AAF, University of Wisconsin - Milwaukee, USA) for their stimulated discussions and invaluable assistance with experiments. This work is supported by NSF-ECS grant 9984225, NIH grants R21RR14257, R15CA83910 and R21RR14257, Research Corporation Award RI0447, and NATO Collaborative Linkage Grant 979419.

\section{REFERENCES}

1 G. Karp, Cell and Molecular Biology, John Wiley and Sons, Inc., 3d edition, 2002.

2 K. Clays, E. Hendrick, M. Triest, T. Verbiest, A. Persoons, C. Dehu, and J. L. Bredas, "Nonlinear optical properties of proteins measured by Hyper-Rayleigh scattering in solution", Science 262, 1419-1422 (1993).

3 G. Cox, E. Kable, A. Jones, I. K. Fraser, F. Manconi, and M. D. Gorrell, "3-dimensional imaging of collagen using second harmonic generation", J. Struct. Biol. 141, 53-62 (2003).

4 D. N. Reinhoudt, and M. Crego-Calama, "Synthesis beyond the molecule", Science 295, 2403-2407 (2002).

5 P. Prasad and D. Williams, Introduction to nonlinear optical effects in molecules and polymers, John Wiley \& Sons, Inc., New York, 1991.

6 S. Roth and I. Freund, "Optical second-harmonic scattering in rat-tail tendon”, Biopolym. 20, 1271-1290 (1981).

7 I. Freund, M. Deutsch, and A. Sprecher, "Optical second-harmonic microscopy, crossed-beam summation, and small-angle scattering in rat-tail tendon”, Biophys. J. 50, 693-712 (1986).

8 Y. Guo, P. Ho, A. Tirksliunas, F. Liu, and R. R. Alfano, "Optical harmonic generation from animal tissues by the use of picosecond and femtosecond laser sources", Appl. Opt. 35, 6810-6813 (1996).

9 E. Georgiou, T. Theodossiou, V. Hovhannisyan, K. Politopoulos, G. Rapti, and D. Yova, Opt. Commun. 176, 253 (2000). 
10 P. Stoller, K. Reiser, P. Celliers, and A. Rubenchik, "Polarization-modulated second harmonic generation in collagen", Biophys. J. 82, 3330-3342, (2002).

11 V. Shcheslavskiy, G. Petrov, and V.V. Yakovlev, "Nonlinear susceptibility measurements of solutions using thirdharmonic generation on the interface", Appl. Phys. Lett. 82, 3982-3984 (2003).

12 G. Bjorklund, "Effects of focusing on third-order nonlinear processes in isotropic media", IEEE J. Quant. Electron. 11, 287-296 (1975).

13 R. W. Boyd, Nonlinear optics, Academic Press, Boston, 1992.

14 A. Kaminska and A. Sionkowska, "Effect of UV radiation on the infrared spectra of collagen", Polym. Degrad. Stabil. 51, 19-26 (1996).

15 V. Shcheslavskiy, G. I, Petrov, S. Saltiel, and V. V. Yakovlev, "Quantitative characterization of aqueous solutions probed by the third-harmonic generation microscopy", J. Struct. Biology (2004) In press. 